\title{
Optical fibers: Interaction dispersion-chirp in directly modulated systems
}

\section{Fibras ópticas: Interacción dispersión-chirp en sistemas ópticos modulados directamente}

\author{
Carmina del Río( $\left.{ }^{(1,}\right)$, Paloma R. Horche(2) \\ 1. Departamento de Sistemas de Ingeniería de Sistemas de Información y Telecomunicación, \\ Universidad CEU San Pablo, Madrid, Spain. \\ 2. Departamento de Tecnología Fotónica y Bioingeniería, ETSI Telecomunicación, \\ Universidad Politécnica de Madrid, Spain. \\ (*) Email: carmina.eps@ceu.es \\ Received / Recibido: 31/01/2013. Revised / Revisado: 10/04/2013. Accepted / Aceptado: 25/04/2013. \\ DOI: http://dx.doi.org/10.7149/OPA.46.3.231
}

\begin{abstract}
:
The directly modulated laser (DML) is usually used in access/metro networks with a bit rate of up to $10 \mathrm{~Gb} / \mathrm{s}$. This configuration has several advantages over external modulation, such as low cost, simplicity of design, small size and high output power. However, the frequency shift of the laser (chirp), associated with the DML, is a serious drawback to be approached in systems using this transmitter model. This work proposes a method for optimizing directly modulated systems. This method determines under what conditions the accumulated chromatic dispersion in the link can be counteracted through proper choice of laser chirp and nonlinear phenomena in optical fiber.
\end{abstract}

Key words: Directly Modulated Laser (DLM), Chirp, Dispersion, Optical Fiber.

\section{RESUMEN:}

En los sistemas de comunicación por fibra óptica, la configuración usualmente empleada para velocidades de transmisión no superiores a $10 \mathrm{~Gb} / \mathrm{s}$ es la modulación directa del láser (DML). Esta configuración presenta diversas ventajas frente a la modulación externa, como son un bajo coste, simplicidad de diseño, tamaño reducido y una elevada potencia de emisión. Sin embargo, el desplazamiento de la frecuencia nominal del láser (chirp) asociado al DML es un grave inconveniente al que deben enfrentarse los sistemas que trabajan con este modelo de transmisor. En este trabajo se propone un método para la optimización de los sistemas modulados directamente. Este método determina bajo qué condiciones se consigue contrarrestar la dispersión acumulada en el enlace ajustando adecuadamente el chirp del láser y los fenómenos no lineales que se generan en la fibra óptica.

Palabras clave: Láser Modulado Directamente, Chirp en Frecuencia, Dispersión Cromática, Fibra Óptica.

\section{REFERENCES AND LINKS / REFERENCIAS Y ENLACES}

[1]. I. Sogawa, N. Kaida, K. Iwai, T.Takagi, T. Nakabayashi, G.Sasaki, "Study on full spectrum directly modulated CWDM transmission of $10 \mathrm{~Gb} / \mathrm{s}$ per channel over water-peak-suppressed non-zero dispersion shifted fiber", 28th European Conference on Optical Communication. ECOC 2002, vol. 3, 1-2 (2002).

[2]. J. D. Downie, M. Sauer, J. Hurley, "40 Gb/s uncompensated 8-channel CWDM system over $30 \mathrm{~km}$ of non-zero dispersion shifted fibre", $31^{\text {st }}$ European Conference on Optical Communication ECOC 2005, vol. 4, 857-858 (2005).

[3] G. P. Agrawal, Fiber-Optic Communication Systems, John Wiley \& Sons (2010). DOI 
[4]. P. J. Corvini, T. L. Koch, "Computer simulation of high bit rate optical fiber transmission using singlefrequency lasers”, J. Lightwave Technol. 5, 1591-1595 (1987). DOI

[5]. I. Tomkos, I. Roudas, R. Hesse, N. Antoniades, A. Boskovic, R. Vodhanel, "Extraction of laser rate equations parameters for representative simulations of metropolitan-area transmission systems and networks", Opt. Commun. 194, 109-129 (2001). DOI

[6]. J. A. P. Morgado, A. V. T. Cartaxo, "Directly modulated laser parameters optimization for metropolitan area networks utilizing negative dispersion fibers", IEEE J. Sel. Top. Quant. 9, 1315-1324 (2003). DOI

[7]. C. H. Henry, "Theory of the linewidth of semiconductor lasers", IEEE J. Quantum Electron. 18, 259264 (1982). DOI

[8]. M. Osinski, J. Buss, "Linewidth broadening factor in semiconductor lasers -An overview", IEEE J. Quantum Elect. 23, 9-29 (1987). DOI

[9]. C. del Río, P. R. Horche, "Directly modulated laser intrinsic parameters: Optimization for WDM systems", International Conference on Advances in Electronics and Micro-electronics, Valencia (2008).

[10]. K. Hinton, T. Stephens, "Specifying adiabatic lasers for 2-5 gbit/s. High dispersion IM/DD optical systems", Electron. Lett. 29, 1479-1480 (1993). DOI

[11]. N. Suzuki, T. Ozeki, "Simultaneous compensation of laser chirp, Kerr effect, and dispersion in 10-Gb/s long-haul transmission systems", J. Lightwave Technol. 11, 1486-1494 (1993). DOI

[12]. I. Tomkos, D. Chowdhury, J. Conradi, D. Culverhouse, K. Ennser, C. Giroux, B. Hallock, T. Kennedy, A. Kruse, S. Kumar, N. Lascar, I. Roudas, M. Sharma, R. S. Vodhanel, C.-C. Wang, "Demonstration of negative dispersion fibers for DWDM metropolitan area networks", IEEE J. Select. Top. Quant. 7, 439460 (2001). DOI

[13]. L.-S. Yan, C. Yu, Y.Wang, T. Luo, L. Paraschis, Y. Shi, A. E. Willner, “40-Gb/s transmission over 25 km of negative-dispersion fiber using asymmetric narrow-band filtering of a commercial directly modulated DFB Laser", IEEE Photon. Technol. Lett. 17, 1322-1324 (2005). DOI

[14]. P. Horche, C. del Río, "Enhanced performance of WDM systems using directly modulated lasers on positive dispersion fibers", Opt. Fiber Technol. 14, 102-108 (2008). DOI

[15].C. del Río Campos, P. R. Horche. "Concatenación de filtros en redes CWDM en anillo que utilizan láseres modulados directamente”, VI Reunión Española de Optoelectrónica - Optoel'09, Libro de Actas, pp. 425-430 (2009).

[16]. P. Krelik, "Characterization of semiconductor laser frequency chirp based on signal distortion in dispersive optical fiber", Opto-electron. Rev. 14, 123-128 (2006).

\section{Introducción}

En los sistemas de comunicación por fibra óptica, la configuración usualmente empleada para velocidades de transmisión no superiores a $10 \mathrm{~Gb} / \mathrm{s}$ es la modulación directa del láser (DML). Esta configuración presenta diversas ventajas frente a la modulación externa, como son un bajo coste, simplicidad de diseño, tamaño reducido y una elevada potencia de emisión. Por otra parte, los espectros de este tipo de fuentes son más anchos y asimétricos que los que provienen de fuentes con modulación externa y además presentan un desplazamiento respecto a su frecuencia nominal al aumentar su potencia de emisión. Este efecto, conocido como chirp en frecuencia óptica, es el principal inconveniente al que se enfrentan los sistemas que trabajan con este modelo de fuente.
La presencia de chirp tendrá diferentes consecuencias sobre la calidad final del sistema según sean las propiedades dispersivas de la fibra óptica por la que se propague. En determinadas condiciones, el chirp del pulso óptico puede compensar la dispersión de la fibra y mejorar el funcionamiento general del sistema.

El objetivo de este estudio es encontrar dicha situación, es decir, convertir un fenómeno que inicialmente se considera pernicioso, como es la presencia de chirp, en una mejora de la calidad. Para la realización de este trabajo se han evaluado de forma teórica y mediante simulación, la interacción entre la dispersión de la fibra y el chirp de los láseres, considerando pulsos ópticos con diferentes tipos de formas. Se propone un método para el diseño de sistemas ópticos que consigue máxima calidad de transmisión y se presentan los resultados 
obtenidos tanto para un sistema monocanal como para sistemas multiplexados en longitud de onda con tecnología CWDM (Coarse Wavelength Division Multiplexing).

\section{Dispersión cromática en fibras}

La dispersión temporal, es decir, el ensanchamiento que sufren los pulsos ópticos al atravesar un medio, es la principal causa de las limitaciones que aparecen en los sistemas de comunicaciones ópticas que trabajan a altas velocidades ya que puede provocar solapamientos entre pulsos contiguos haciendo irreconocible la información que llega al receptor.

En el caso de fibras monomodo, el ensanchamiento de los pulsos se debe principalmente a la dependencia que existe entre la velocidad de grupo a la que se propaga la información y la longitud de onda. Esta dependencia implica que las distintas frecuencias ópticas que configuran el espectro de la señal viajen a diferentes velocidades, alcanzando al receptor en momentos diferentes y provocando en los pulsos ópticos una dispersión en el tiempo. A este fenómeno se le conoce como dispersión cromática y se caracteriza mediante el coeficiente de dispersión cromática, $D(\mathrm{ps} / \mathrm{nm} \cdot \mathrm{km})$.

Existe una longitud de onda $\left(\lambda_{Z D}\right)$ para la cual el coeficiente de dispersión cromática de la fibra se hace nulo $(D=0)$ y representa un punto de inflexión en el estudio del comportamiento de los sistemas que trabajan a longitudes de onda superiores e inferiores a este valor. De este modo, en régimen de dispersión normal, es decir, si se trabaja en el rango de longitudes de onda con un coeficiente de dispersión negativo $(D<0)$, las longitudes de onda más largas ('red end') de un pulso libre de chirp viajarían más rápidas que las longitudes de onda más cortas, por lo que el pulso se estrechará tras su recorrido por la fibra. Este efecto se suele denominar como chirp positivo.

En régimen de dispersión anómala $(D>0)$, las longitudes de onda más cortas ('blue end') viajarán más rápidas que las largas, por lo que después de recorrer la fibra, el pulso óptico se ensanchará en el tiempo (chirp negativo).

Variando la distribución del perfil del índice de refracción del núcleo se puede desplazar la longitud de onda de dispersión nula y conseguir distintos modelos de curvas de dispersión.

El sector de estandarización de Telecomunicaciones de la ITU define, en las recomendaciones ITU-T G-SERIES, cuatro categorías de fibras monomodo en las que la principal característica que las diferencia es su dispersión cromática:

- ITU-T G.652: Characteristics of a single-mode optical fibre (11/2009),

- ITU-T G.653: Characteristics of a dispersionshifted single-mode optical fibre and cable (07/2010),

- ITU-T G.655: Characteristics of a non-zero dispersion shifted single-mode optical fibre and cable (11/2009),

- ITU-T G.656: Characteristics of a fibre and cable with non-zero dispersion for wideband optical transport (07/2010).

Las fibras a las que hace referencia la Recomendación G.652, se las suele denominar fibra monomodo estándar (SMF). Es la más ampliamente utilizada y existen millones de $\mathrm{km}$ de este tipo de fibra instalados en redes ópticas de todo el mundo, que se benefician de sus bajas pérdidas a $1550 \mathrm{~nm}$ y de la posibilidad de utilización de los amplificadores ópticos de fibra dopada con erbio (EDFA). Debido a la evolución de los requerimientos de las redes ópticas, las Recomendaciones de la ITU han ido adaptándose a ellas y, desde la primera versión publicada en 1984 hasta la última en noviembre de 2009, se han contemplado mejoras en las características de estas fibras, desdoblándose en cuatro categorías entendiendo a la atenuación en la Banda $\mathrm{E}$ y a la exigencia de menor dispersión por modo de polarización (PMD), en determinadas aplicaciones.

El coeficiente de dispersión cromática de la G.652, para longitudes de onda en torno a 1550 $\mathrm{nm}$, es de aproximadamente $17 \mathrm{ps} / \mathrm{km}-\mathrm{nm}$, lo que permite su utilización en sistemas DWDM con gestión de la dispersión y facilita la no 
aparición de efectos no lineales. Algunos ejemplos de este tipo de fibra son: SMF-28 (Corning) y AllWave (Lucent). Esta última se caracteriza por eliminar el pico de absorción del ión $\mathrm{OH}^{-}$, por lo que ofrece un mayor espectro para la transmisión en sistemas multicanal CWDM.

Para hacer coincidir el mínimo de atenuación con el mínimo de dispersión, se creó la fibra de dispersión desplazada (DSF), con una longitud de onda de dispersión nula a $1550 \mathrm{~nm}$, cuyas especificaciones se recogen en la Recomendación G.653. En este tipo de fibra se refuerzan los efectos de los fenómenos no lineales en sistemas DWDM en la banda C, por lo que se desarrolló otro modelo, denominado fibra de dispersión desplazada no nula (NZ-DSF), G.655, que se caracteriza por valores del coeficiente dispersión cromática reducidos pero no nulos en toda la banda C, positivos (NZDSF+) o negativos (NZDSF-). La fibra G.656 es similar a la G.655 pero con el coeficiente dispersión siempre positivo (NZDSF+) en todo el rango de frecuencia ópticas de la banda C. Esto permite la gestión de la dispersión acumulada intercalando fibras compensadoras de la dispersión de coeficiente negativo. Las fibras G.655 y G.656 han sido las más instaladas en los últimos años, sobre todo en enlaces transoceánicos y troncales de larga distancia, aunque también han despertado interés en aplicaciones CWDM [1,2]. Algunos ejemplos de estas fibras comerciales son: LEAF® y MetroCor (Corning), TrueWave ${ }^{\circledR}$ RS (Lucent/OFS) y Teralight (Alcatel).

En la Fig. 1 se muestran los perfiles típicos de dispersión (a) y de atenuación (b) para estos modelos de fibras.

\section{Fuentes moduladas directamente}

La modulación en amplitud de una fuente óptica se puede llevar a cabo con un diodo láser (LD) emitiendo en continua al que se añade un modulador externo o modulando directamente el láser a través de su corriente de polarización.

Los láseres modulados externamente constan de un generador de bits $\left(a_{k}\right)$, un generador de pulsos eléctrico, con codificación RZ (Return to

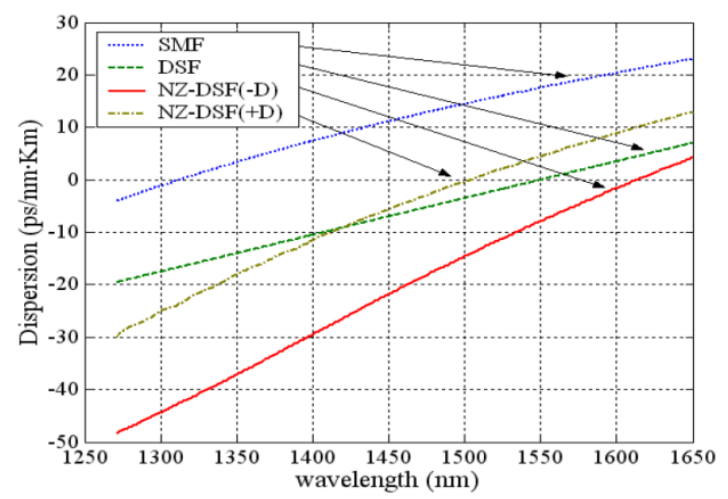

(a)

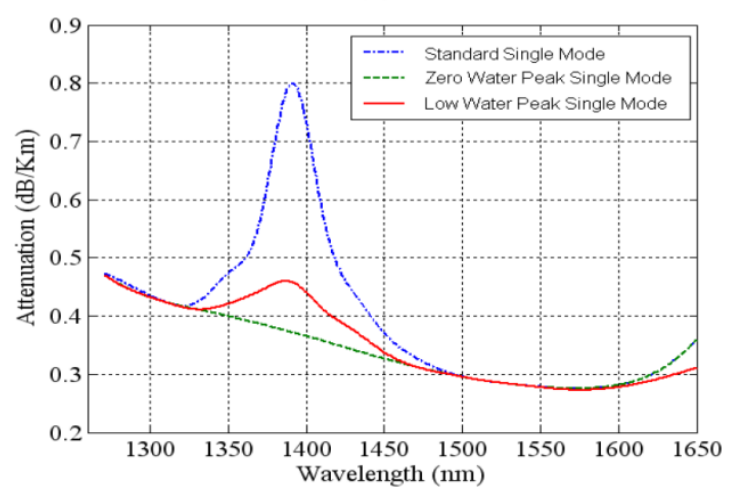

(b)

Fig. 1. Curvas de dispersión (a) y de atenuación (b) en función de la longitud de onda para diferentes modelos de fibra óptica.

Zero) o NZR (No Return to Zero), un láser LDDFB y un modulador externo, normalmente tipo Mach-Zender, al que se le aplica directamente la tensión de modulación, como se muestra en la Fig. 2.

La secuencia de bits transmitidos $a_{k}$ $\left(a_{k}=1(0)\right.$ para el envío de un uno (cero) lógico) durante un tiempo $k$ se convierte en una sucesión de pulsos eléctricos con un periodo de modulación $T$, que son superpuestos sobre una corriente de polarización $I_{b}$ (bias current). El resultado es una señal de corriente eléctrica que ataca a los electrodos de control del modulador externo y que se puede expresar según la ecuación:

$$
I(t)=I_{b}+\sum_{k=-\infty}^{+\infty} a_{k} I_{p}(t-k T),
$$

donde $I_{p}(t)$ es el pulso de corriente aplicado. 


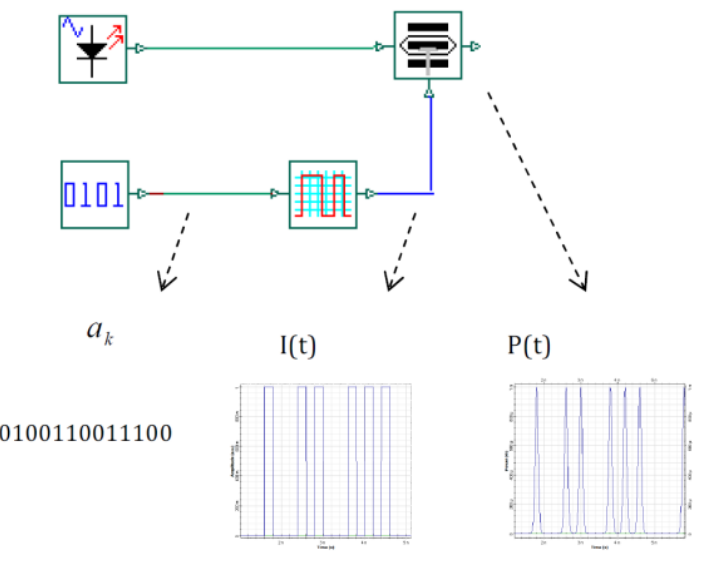

Fig. 2. Diagrama de bloques de una fuente modulada externamente.

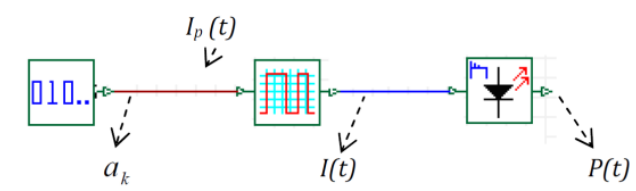

0100110011100
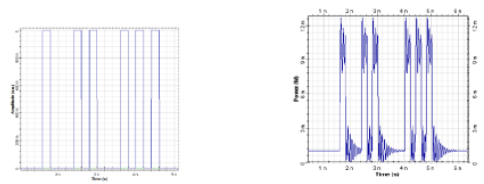

Fig. 3. Diagrama de bloques de una fuente modulada directamente.

Sin embargo, en diversos ámbitos como por ejemplo en las redes metropolitanas y de acceso suelen emplearse láseres modulados directamente (DML), debido a su bajo coste y a sus pequeñas dimensiones. La Fig. 3 representa el diagrama de bloques de una fuente modulada directamente, que en el caso más sencillo supone el encendido o apagado de la fuente con cada 1 ó 0 enviado.

Si se considera una fuente ideal, con modulación externa, la potencia óptica a la salida del láser $P(t)$ puede determinarse directamente a partir de la corriente $I(t)$ [3], es decir, variará linealmente con la energía de los fotones a la frecuencia óptica de emisión ( $h v$ ) y con la eficiencia cuántica diferencial del láser $\left(\eta_{0}\right)$ tal y como queda recogido en la siguiente ecuación:

$$
P(t)=\eta_{0} \frac{h v}{q} \sum_{k=-\infty}^{+\infty} a_{k}(t-k T) .
$$

Sin embargo, la expresión (2) no se puede aplicar en el caso de fuentes moduladas directamente. En este tipo de fuentes, en las que se inyecta una corriente eléctrica variable directamente sobre el circuito de alimentación del láser, se produce una variación en el número de portadores de la región activa que altera el valor del índice de refracción, $\Delta n$. Esta variación origina a su vez variaciones en la fase óptica de la señal, $\Delta \phi(t)$, y por tanto en la frecuencia de emisión, $\Delta v(t)$.

La variación de frecuencia óptica producida a lo largo del pulso emitido, conocido como chirp, es una característica típica de los láseres modulados directamente y puede expresarse como:

$$
\Delta v(t)=\frac{1}{2 \pi} \frac{d \phi}{d t} .
$$

En los siguientes subapartados se analiza la evolución del chirp a lo largo de un pulso óptico. Esta evolución dependerá de la forma del pulso por lo que se estudian diferentes casos. En primer lugar se supone una forma de pulso Gaussiana que debido a su sencillez nos permitirá entender mejor el proceso y finalmente generalizarla a una forma de pulso arbitraria.

\section{3.a. Pulsos ópticos con forma Gaussiana}

En esta primera sección se considera que el pulso presenta una forma Gaussiana. Esta consideración se debe a que las funciones Gaussianas suponen un modelo matemático fácil de usar y además la transformada de Fourier de funciones Gaussianas son también funciones Gaussianas.

En la Fig. 4(a) se muestra un pulso óptico de estas características sobre el que se ha superpuesto la variación en la fase óptica que se produce a lo largo del mismo.

Dado que el desplazamiento en frecuencias o chirp se calcula derivando la fase óptica en (3) y que ésta tiene forma cuadrática, se deduce que el chirp asociado a este tipo de fuentes será una función lineal (Fig. 4(b)).

Una variación de frecuencia lineal se puede expresar matemáticamente como $f(t)=f_{0}+C t$, donde $f_{0}$ sería la frecuencia inicial (valor de la frecuencia para un punto determinado que consideraremos en $t=0$ ) y $C$ representa el incremento de frecuencia a lo largo del pulso o chirp. Si $C$ es positivo, la frecuencia en el pulso 

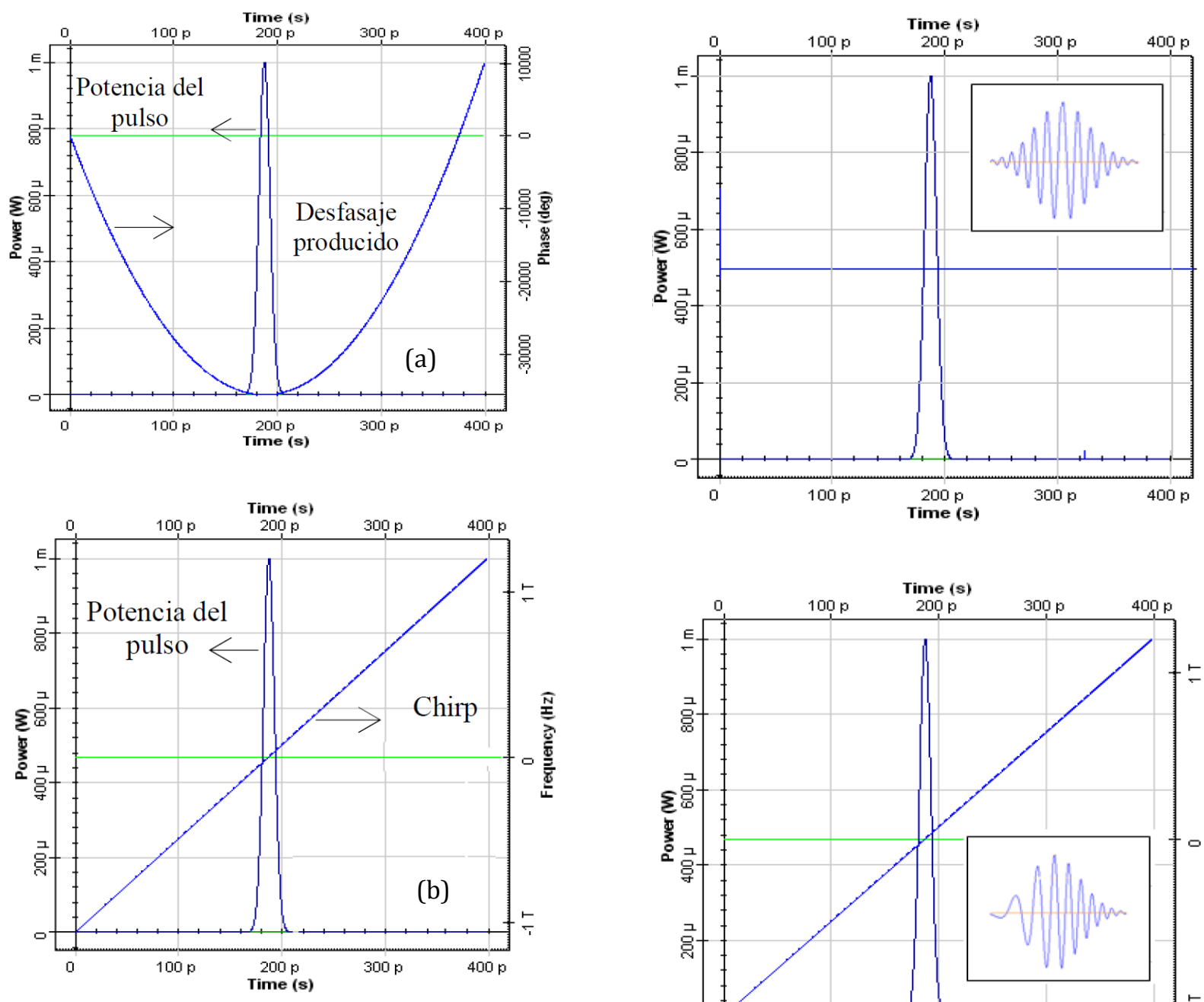

Fig. 4. Potencia, desfasaje y chirp de un pulso óptico: (a) Intensidad de pulso y desfasaje. (b) Intensidad de pulso y chirp.

aumentará linealmente con el tiempo, por el contrario si es negativo, disminuirá también de forma lineal.

Es decir, en un pulso Gaussiano libre de chirp, la frecuencia óptica se mantiene constante a lo largo de todo el pulso (Fig. 5(a)), mientras que en un pulso con un ratio de chirp positivo/negativo la desviación de la frecuencia se podría representar como una línea de pendiente positiva/negativa, tal y como muestran las Figs. 5(b) y 5(c).

\section{3.b. Pulsos ópticos con forma arbitraria}

En los sistemas modulados directamente, la variación de la corriente de modulación del láser provoca que la forma del pulso óptico generado no sea una réplica de la forma del pulso eléctrico
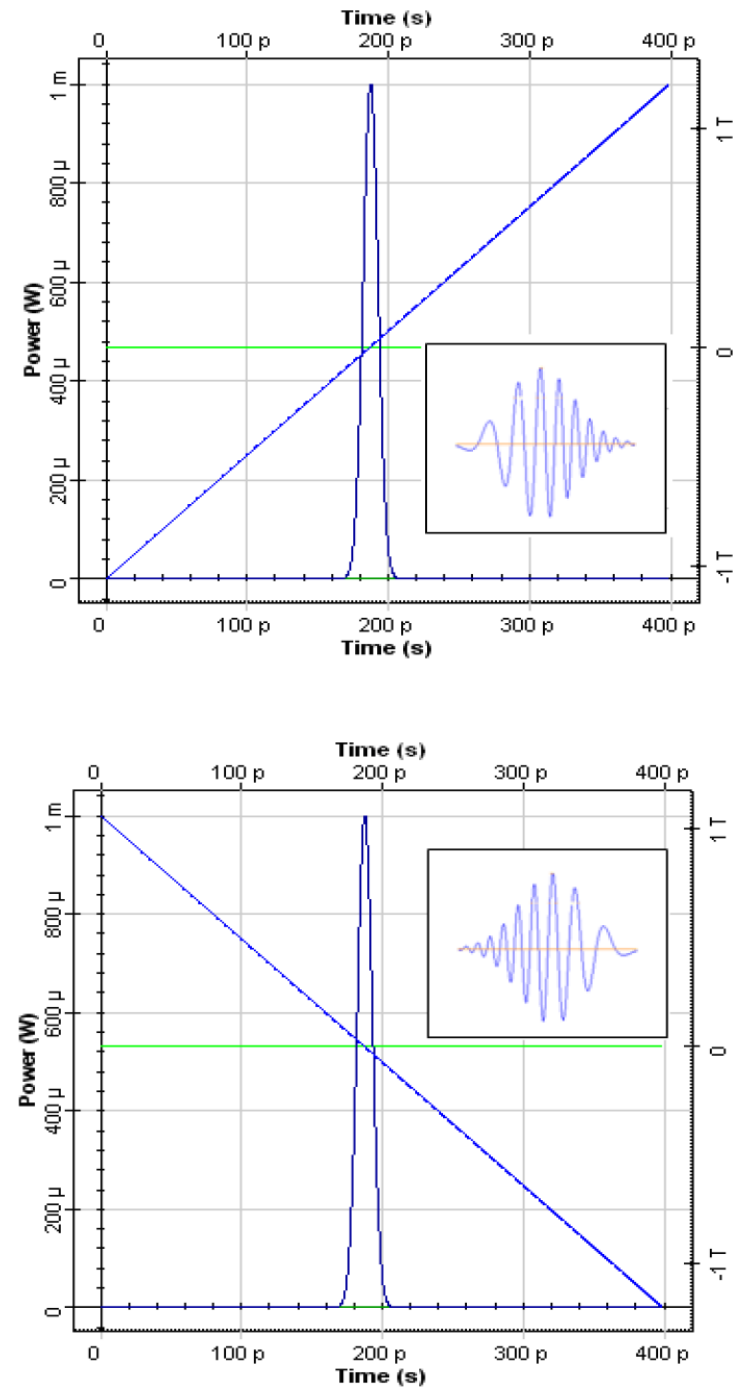

Fig. 5. Pulso, chirp y frecuencia óptica de los pulsos ópticos para tres señales diferentes: (a) Fuente libre de chirp. (b) Fuente con chirp positivo. (c) Fuente con chirp negativo. 


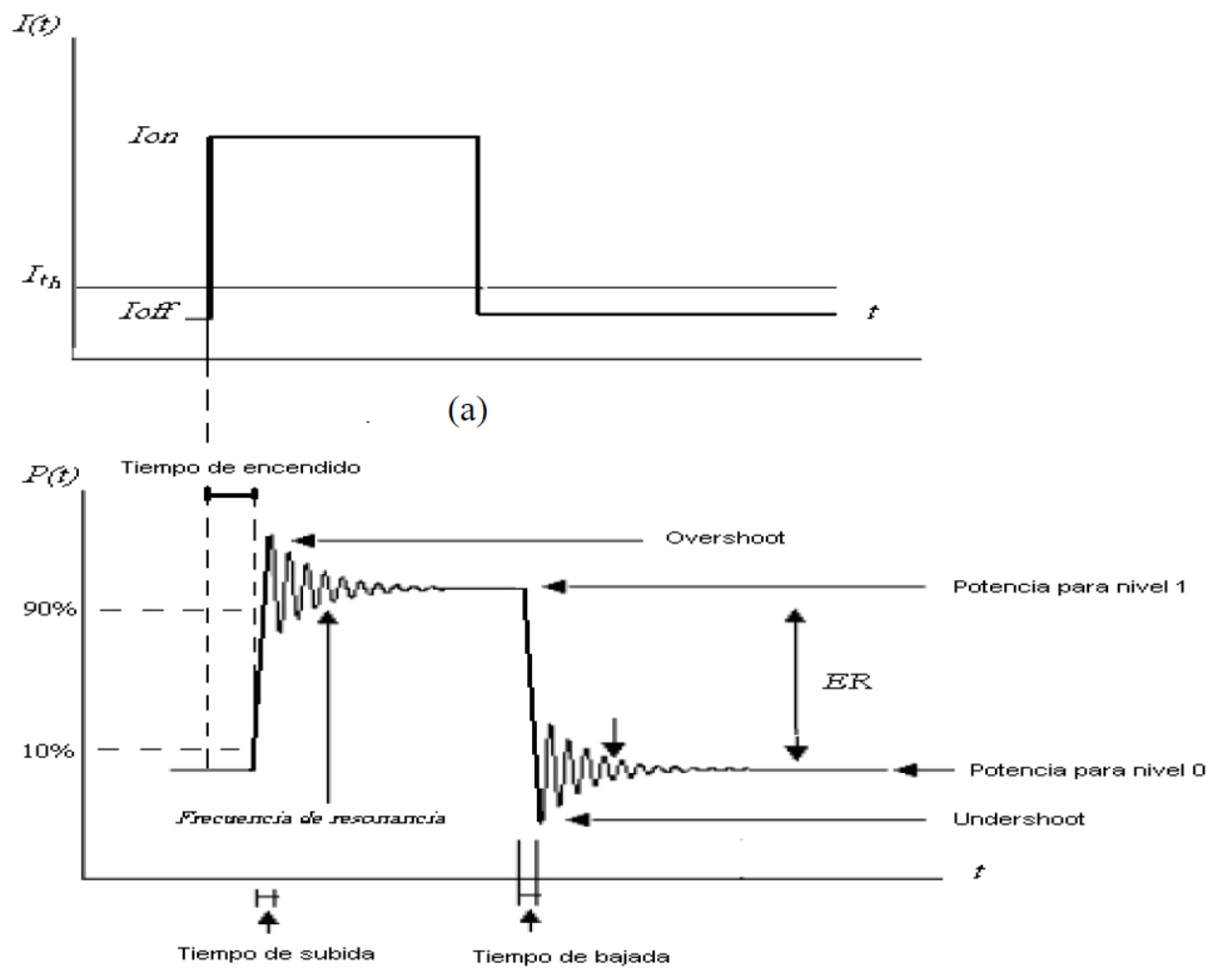

(b)

Fig. 6. Forma del pulso óptico $P(t)$ al ser excitado por una función escalón $I(t)$.

de modulación. En concreto, al aplicar al láser un escalón de corriente (Fig. 6(a)), la potencia óptica de salida del láser adquirirá una forma similar a la mostrada en la Fig. 6(b).

Debido a la lenta respuesta de los portadores de carga, el láser tardará un tiempo en alcanzar la concentración de portadores en transparencia. A este tiempo se le denomina tiempo de encendido, $t_{D}$.

Una vez alcanzada la concentración de portadores umbral, el pulso óptico tardará un tiempo en alcanzar su valor final. El tiempo que tarda la potencia óptica en pasar del 10\% al 90\% de su valor máximo se denomina tiempo de subida y se define de forma análoga el tiempo de bajada (90\%-10\%).

Por otra parte la estabilización de la potencia óptica emitida por el láser tampoco ocurre de forma inmediata. Al comienzo y al final de cada pulso óptico aparecen ciertas oscilaciones amortiguadas (oscilaciones de relajación) cuyo origen está en el proceso por el que los portadores y fotones tienden a sus valores de equilibrio. El overshoot y undershoot representan los niveles de potencia que sobrepasan los valores medios asignados para el envío de unos y ceros, respectivamente.

En estos casos, en el que las variaciones de fase dejan de ser cuadráticas, la desviación en frecuencia deja de ser lineal y, por tanto, ya no puede caracterizarse mediante el valor de la constante de chirp (C). Corvini y Koch dedujeron una expresión válida para el cálculo del chirp al trabajar con láseres modulados directamente construidos a partir de láseres de semiconductor del tipo Fabry-Perot [4] y posteriormente se demostró que podía ser utilizada también como una buena aproximación para láseres del tipo DFB [5,6]. Dicha expresión, siempre que se trabaje con frecuencias de modulación mayores a $100 \mathrm{MHz}$, depende fundamentalmente del coeficiente de chirp adiabático del láser $\kappa$ y el factor de Henry $\alpha$ a través de la siguiente ecuación: 


$$
\begin{aligned}
\Delta v(t)=\frac{\alpha}{4 \pi}\left(\frac{1}{P(t)} \frac{d P(t)}{d t}+\frac{P(t) \varepsilon}{\tau_{p}}\right)= \\
=\frac{\alpha}{4 \pi}\left(\frac{1}{P(t)} \frac{d P(t)}{d t}+\kappa P(t)\right) .
\end{aligned}
$$

El coeficiente adiabático $\kappa$ es un parámetro que depende de la estructura del láser y que puede expresarse en función de la energía del fotón a la frecuencia óptica de emisión, $h v$, de la eficiencia cuántica del láser $\left(\eta_{0}\right)$, del factor de confinamiento $(\Gamma)$, del volumen de la cavidad activa $\left(V_{a}\right)$ y del factor de compresión $(\varepsilon)$.

$$
\kappa=\frac{2 \Gamma}{\eta_{0} h v V_{a}} .
$$

Sus unidades de medida son $(\mathrm{W} \mathrm{S})^{-1}$ y toma valores del orden de $10^{12}$.

El factor de Henry $(\alpha)$, también llamado factor de ensanchamiento de línea, representa la derivada de la parte real del índice de refracción complejo respecto de la parte imaginaria. Caracteriza la propagación por el medio activo o, lo que es lo mismo, las variaciones que se producen en el índice de refracción $(d n)$ y en la ganancia $(d g)$ cuando se modifica la densidad de los portadores $d N$ :

$$
\alpha=-\frac{\partial \chi_{r} / \partial N}{\partial \chi_{i} / \partial N}=-\frac{4 \pi}{\lambda} \frac{d n}{d g} .
$$

El factor de Henry puede tomar valores comprendidos entre 2 y 9 según el tipo de material que se emplee para configurar la capa activa del láser [7] y es de vital importancia a la hora de parametrizar el DML ya que determina su ancho de línea, el valor del chirp y su respuesta a la realimentación óptica [8].

El primer término de (4) es independiente de la estructura interna del láser y caracteriza el chirp que se produce en las transiciones de 0 a 1 y de 1 a 0 , por lo que se denomina chirp transitorio y está ponderado por el parámetro $\alpha$ [9].

El segundo término de (4) depende de la estructura del láser y se denomina chirp adiabático. Este chirp es el responsable del desplazamiento del espectro hacia el azul cuando se emiten altos valores de potencia y de las asimetrías típicas en los espectros de estas fuentes. Esta terminología la empleó por primera vez Hinton [10] en 1993.
Dependiendo del término de (4) que predomine sobre el otro, se puede hacer una clasificación de los DML, llamando DML con comportamiento transitorio dominante (DML-T) a aquellos en los que predomina el chirp transitorio y DML con comportamiento adiabático dominante (DML-A), aquellos en los que predomina el segundo término.

Existen diferencias sustanciales entre los espectros y las formas de los pulsos ópticos de los transmisores DML-T y DML-A. Los DML-T presentan anchos de banda superiores a los DML-A y cuentan con espectros más asimétricos, tal y como se puede apreciar en la Fig. 7.
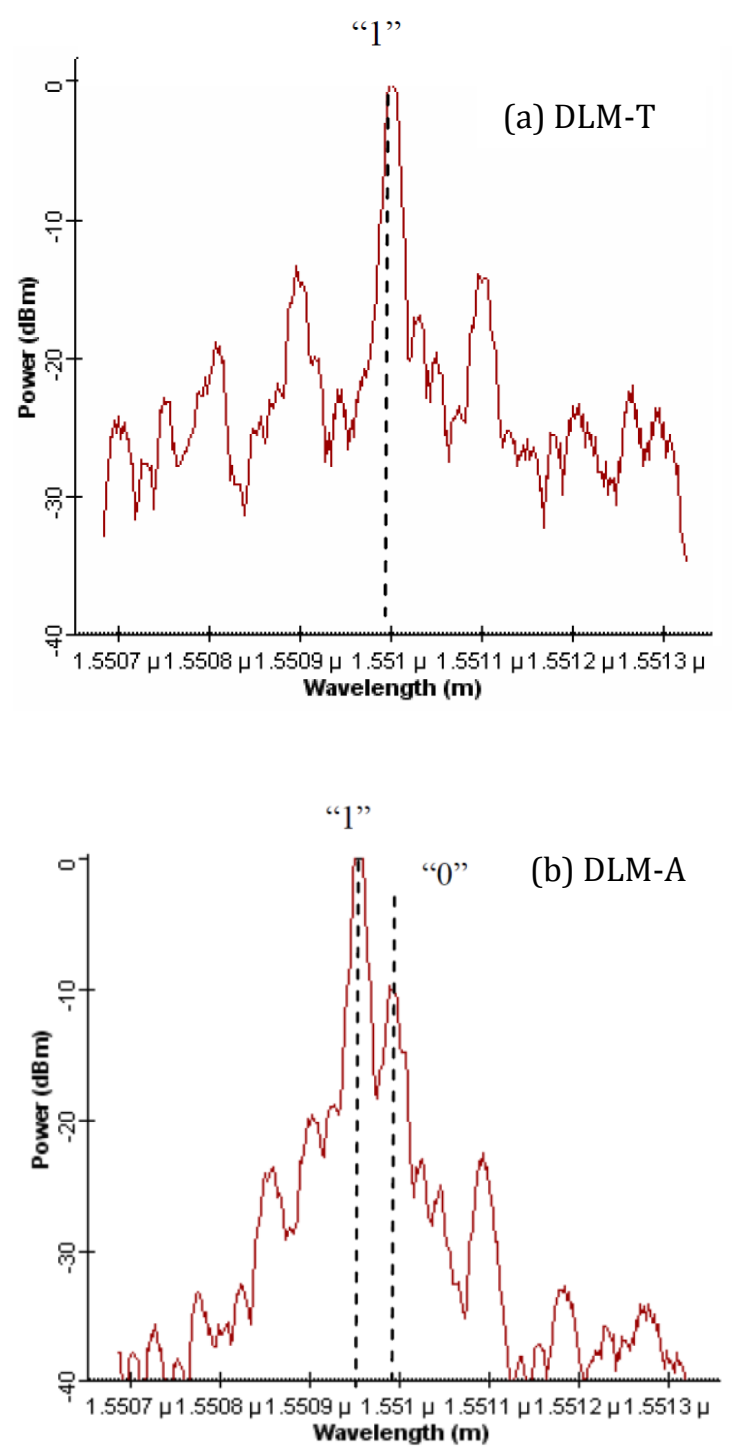

Fig. 7. Espectro de un láser con un comportamiento dominante (a) transitorio y (b) adiabático obtenido mediante simulación. 

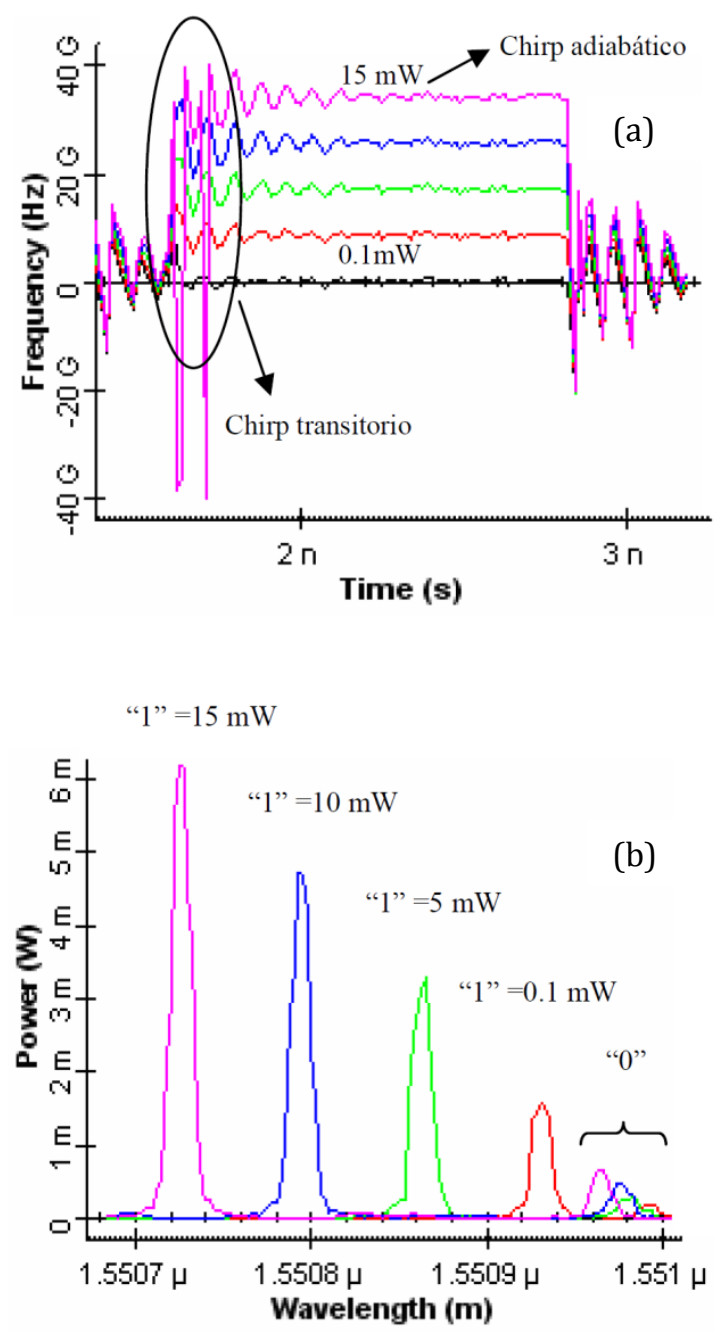

Fig. 8. Chirp (a) y espectro (b) a la salida de un láser con un comportamiento adiabático dominante para varios valores de potencias comprendidos en el rango de 0.1 a $15 \mathrm{~mW}$.

Una de las consecuencias del chirp adiabático es la separación entre las longitudes de onda de las portadoras de los bits 0 y 1 y que, según (4) depende de la potencia óptica emitida $P(t)$.

La Fig. 8(a) se ha obtenido mediante simulación el chirp de un bit "1" cuando la potencia media de emisión del DML varía entre 0.1 a $15 \mathrm{~mW}$. Se observa, por una parte, un chirp transitorio que aparece únicamente al producirse el cambio de estado (de 0 a 1) y, por otra parte, un chirp adiabático que provoca un desplazamiento del espectro hacia el azul proporcional a la potencia óptica (Fig. 8(b)).

\section{Interacción entre el chirp del DML, la dispersión de la fibra y fenómenos no lineales}

Como se ha comentado en la Sección 1, el objetivo final de este trabajo es aprovechar el chirp generado por el DML con el fin de optimizar la calidad de transmisión de un sistema de comunicaciones ópticas modulado directamente. Para ello es necesario un manejo óptimo de la interacción producida entre el chirp, la dispersión cromática y efectos no lineales en la fibra óptica. Por lo tanto, en este estudio habrá que distinguir entre DMLs con diferentes características de chirp (adiabático o transitorio) y fibras con distintas características de dispersión cromática (positiva o negativa).

Para una mejor comprensión del método propuesto en las Secciones 5 y 6, volvamos por un momento a considerar formas de pulsos Gaussianos, ya que nos permitirá, de una forma sencilla, formular matemáticamente todos los procesos involucrados y entender la esencia del procedimiento utilizado mediante la simulación de la propagación del pulso a través de la fibra.

La expresión de un pulso Gaussiano cuyo valor máximo se sitúa inicialmente en $t=0$, con anchura $T_{0}$ y chirp $C$, se puede formular matemáticamente según la expresión:

$$
A(0, t)=A_{0} \exp \left(-\frac{1}{2}(1+i C)\left(\frac{t}{T_{0}}\right)^{2}\right) .
$$

Si el pulso atraviesa un medio dispersivo, su evolución en función de la distancia recorrida se expresa mediante [3]:

$$
\begin{aligned}
A(z, t)=\frac{A_{0}}{\sqrt{b_{f}}} \exp \left[-\frac{\left(1+i C_{1}\right) t^{2}}{2 T_{0} b_{f}^{2}}+\right. & \\
& \left.+\frac{i}{2} \tan ^{-1}\left(\frac{z}{1+C z}\right)\right] .
\end{aligned}
$$

En concreto, cuando el pulso atraviesa una fibra óptica, cuyas características dispersivas vienen determinadas por el parámetro de dispersión de la velocidad de grupo $\mathrm{GVD} \equiv \beta_{2}\left(\beta_{2}=\right.$ $\left.-2 \pi c / \lambda^{2} D\right)$, siendo $c$ la velocidad de la luz, l la longitud de onda y $D$ el coeficiente de dispersión), el ancho del pulso inicial $T_{0}$ tomará un nuevo valor $T_{1}$. En función de estos parámetros se puede definir el factor de 
ensanchamiento del pulso óptico al atravesar una distancia $z\left(b_{f}\right)$ como:

$$
b_{f}(z)=\frac{T_{1}(z)}{T_{0}(z)}=\sqrt{\left(1+\frac{C \beta_{2} z}{T_{0}^{2}}\right)^{2}+\left(\frac{\beta_{2} z}{T_{0}^{2}}\right)^{2}} .
$$

La evolución del chirp a través del pulso, también se verá alterado y variará de un valor inicial $C$ a otro $C_{1}$ según la ecuación:

$$
C_{1}(z)=C+\left(s\left(1+C^{2}\right)\right) z .
$$

siendo $s$ el signo que toma el parámetro $\beta_{2}$.

Es decir, el pulso Gaussiano seguirá siendo Gaussiano al propagarse a través de la fibra óptica pero su anchura $T_{0}$ y su chirp $C$ variarán en función de la distancia recorrida $z$ y de las características dispersivas de la fibra $\beta_{2}$.

Si el pulso inicial está libre de chirp $(C=0)$ éste se ensanchará monótonamente a su paso a través de la fibra, como se muestra en la Fig. 9, mientras que los pulsos con chirp distinto de 0 podrían ensancharse o comprimirse en función de los signos de $\beta_{2}$ y de $C$.

Si consideramos que $C$ es el valor inicial del chirp introducido por la fuente, para $\beta_{2} C>0$, el pulso Gaussiano con chirp se ensanchará monótonamente con un factor de ensanchamiento mayor que el de un pulso libre de chirp. La razón es que el chirp inducido por la dispersión se suma al chirp generado por el láser, ya que las dos contribuciones son del mismo signo.

Sin embargo, para $\beta_{2} C<0$ la contribución del chirp inducido por la dispersión de la fibra es de signo opuesto al chirp generado por el láser y, a una distancia determinada, ambas contribuciones podrían cancelarse. En concreto, esto ocurriría cuando el pulso recorra una distancia $\xi=|C| /\left(1+C^{2}\right)$. Bajo esta condición,

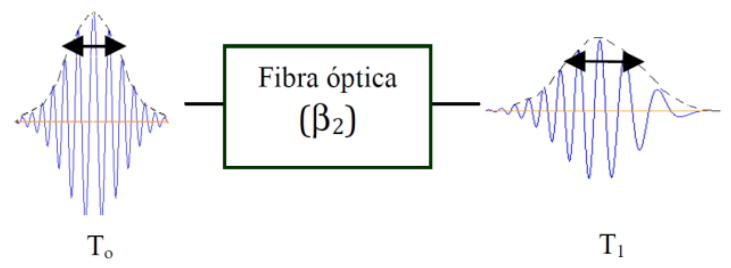

Fig. 9. Pulso óptico libre de chirp al atravesar un medio dispersivo. se obtiene una anchura del pulso mínima $T_{1}^{\min }$ :

$$
T_{1}^{\min }=\frac{T_{0}}{\sqrt{1+C^{2}}} .
$$

Para distancias superiores a $\xi$, el pulso sufrirá un proceso de ensanchamiento pudiendo llegar a ser superior en magnitud al de entrada. Es decir, al igual que un rayo focalizado tiende a la anchura mínima antes de divergir, un pulso con chirp puede reducir su anchura antes de empezar a ensancharse monótonamente.

La Fig. 10 muestra los resultados de la simulación de la evolución de un pulso Gaussiano con chirp positivo que se propaga sobre una fibra de dispersión negativa (Fig. 10(a)). Se representa en las gráficas el chirp asociado (arriba) y la fase (abajo) para distintos puntos del trayecto.

Se puede observar que el pulso adquiere su mínimo ancho cuando ha recorrido una distancia $L_{2}=\xi$ (Fig. 10(b)). En este caso el chirp del láser se ha compensado con la dispersión acumulada a la lo largo de la fibra, consiguiendo un chirp y un desfasaje acumulado nulos y por tanto, las mejores condiciones para la transmisión. A partir de dicho valor el chirp cambiaría de signo e iría aumentando en valor absoluto según avanza a través de la fibra, por lo que el pulso comenzará a ensancharse (Fig. 10(c)).

Para completar este análisis hay que considerar otros fenómenos que puedan modificar el mapa de dispersión del enlace, interviniendo en el balance óptimo entre el chirp de la fuente y la dispersión en fibra. Dentro de esta categoría se encuentran algunos efectos no lineales de la fibra. Tal vez el más destacable sea el fenómeno no lineal conocido como automodulación de fase, SPM.

Como es sabido, el SPM se debe a la dependencia de la fase del campo óptico con la intensidad del pulso propagado. Al transmitir una intensidad modulada en el tiempo, se genera una modulación gradual de la frecuencia (chirp) que hace que cada zona del pulso tenga una frecuencia óptica distinta que a su vez dependerá de la forma del pulso. Por tanto, si la propagación en fibra lleva asociada la presencia de este fenómeno, el chirp debido al SPM se debe contabilizar en el balance óptimo perseguido. 

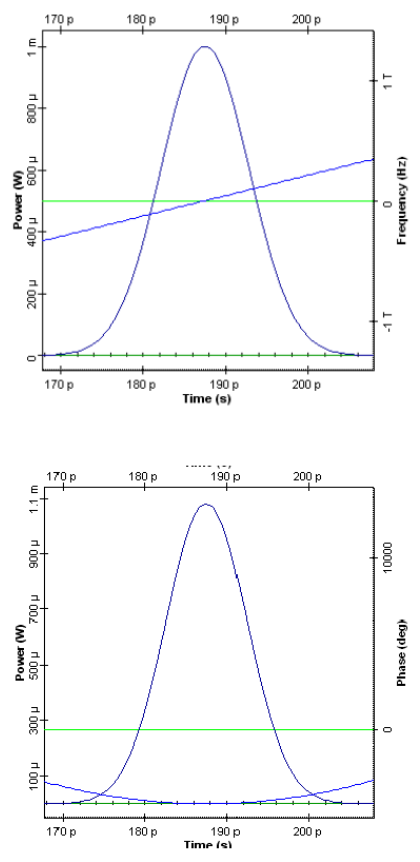

(a) $\mathrm{L}_{1}$
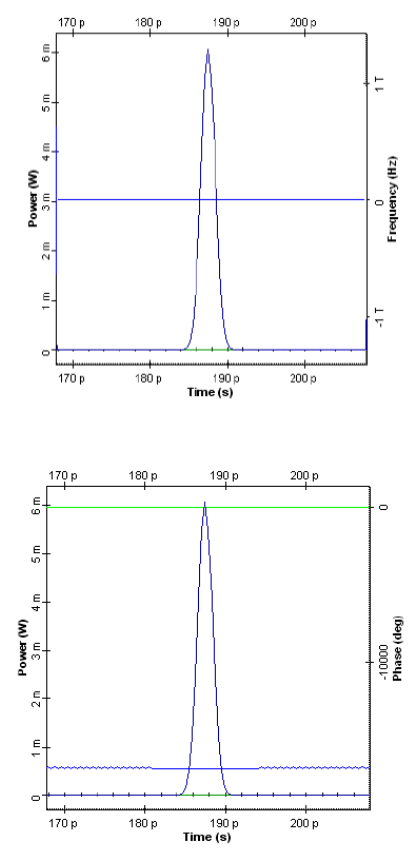

(b) $\mathrm{L}_{2}$
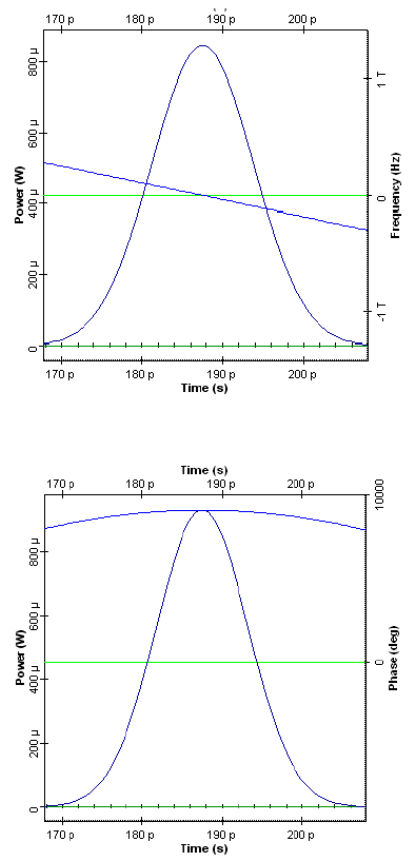

(c) $\mathrm{L}_{3}$

Fig. 10. Chirp (figuras superiores) y desfasaje (inferior) para diferentes distancias de enlace $\left(L_{1}, L_{2}\right.$ y $\left.L_{3}\right)$.

A modo de ejemplo ilustrativo, la Fig. 11 muestra, cualitativamente, el comportamiento un pulso con chirp transitorio dominante en comparación con un pulso afectado por el fenómeno no lineal SPM, al propagarse por un medio con dispersión normal y anómala. Se puede observar que el efecto que se produce en el pulso que proviene de una fuente con un comportamiento transitorio dominante al atravesar un medio dispersivo es el contrario al ocasionado por el efecto no lineal SPM [11].

\begin{tabular}{|c|c|c|}
\hline & $\begin{array}{l}\text { Láser con } \\
\text { chirp transitorio }\end{array}$ & SPM \\
\hline $\begin{array}{l}\text { Pulso de } \\
\text { entrada }\end{array}$ & & \\
\hline Chirp & & $\Delta \lambda$ \\
\hline $\begin{array}{l}\text { Dispersión } \\
\text { normal }\end{array}$ & & ${ }^{P} \uparrow$ \\
\hline $\begin{array}{l}\text { Dispersión } \\
\text { anómala }\end{array}$ & $P \dagger$ & $\mathbf{P}_{\uparrow}$ \\
\hline
\end{tabular}

Fig. 11. Relación entre el chirp transitorio del láser y el SPM.
Teniendo en cuenta que la magnitud del efecto SPM se puede modificar mediante el control de la intensidad óptica propagada en fibra y que según (4), el chirp del DML depende de la potencia de salida del láser, en las siguientes secciones se propone compensar dichos efectos mediante la gestión adecuada de la potencia óptica emitida por el DML y propagada por la fibra. Este método es aplicable a cada uno de los canales de un sistema WDM, como se demuestra en la Sección 6.

\section{Optimización de sistemas sobre fibras de dispersión positiva o negativa}

A lo largo de los últimos años se han propuesto diferentes técnicas para optimizar las redes ópticas metropolitanas que emplean láseres modulados directamente. Algunas de estas propuestas se basan en la sustitución de la fibra convencional SMF por la NZ-DSF [12], que tiene un coeficiente de dispersión negativo en la banda-C, por lo que es capaz de compensar el chirp generado por los láseres modulados directamente; otras proponen el uso de filtros o de circuitos compensadores electrónicos que 
disminuyan el efecto del chirp sobre la transmisión [13 ]. Todas ellas suponen una modificación en el tendido más o menos compleja que, en enlaces ya instalados, podrían ser inviables por el coste económico que supondrían.

En este trabajo se propone otra técnica alternativa que consigue mejorar las condiciones de transmisión sin necesidad de modificar el tendido. Esta propuesta se basa en una correcta elección del tipo de transmisor a emplear y una gestión eficiente de la potencia óptica $P(t)$ emitida que permita una compensación adecuada de la dispersión acumulada en todo el trayecto mediante el balance entre el chirp, dependiente de $P(t)$, la dispersión cromática y las no linealidades que también dependen de $P(t)$. Para analizar bajo qué condiciones se consiguen optimizar sistemas ya instalados, se ha propuesto la simulación de un enlace punto a punto con tecnología CWDM, cuyo esquema se muestra en la Fig. 12. El motivo de analizar un sistema WDM en vez de un sistema monocanal, es debido a la influencia que tiene la función de transferencia óptica de los filtros internos con los que se construyen los multiplexores sobre la señal óptica transmitida. Este estudio estaría incompleto si no se consideran todos los elementos con función selectiva en longitud de onda. Por otro lado, la elección de la tecnología CWDM se basa en que es en este ámbito dónde son usualmente empleados los transmisores DML.
Se han seleccionado 16 canales con longitudes de onda de acuerdo con la Recomendación ITU-T G. 694.2. El pulso se ha codificado con $2^{15}-1$ (OC-48 a $2.5 \mathrm{~Gb} / \mathrm{s}$ ), sin retorno a cero (NRZ) y se han empleado $100 \mathrm{~km}$ de fibra SMF o bien del tipo NZ-DSF.

Para simular el transmisor, se han modelado dos láseres que se corresponden con dispositivos disponibles comercialmente. El denominado DML-A, tiene un comportamiento adiabático dominante (factor de Henry de $\alpha=2.2$, coeficiente adiabático $\kappa=28.7 \times 10^{12}$ $(\mathrm{W} \cdot \mathrm{s})^{-1}$ ) y el DML-T, un chirp transitorio dominante $\left(\alpha=5.6\right.$ y $\left.\kappa=1.5 \times 10^{12}(\mathrm{~W} \cdot \mathrm{s})^{-1}\right)$. A partir de estos componentes, realizando todas las combinaciones posibles entre ellos, se han generado 4 conjuntos de sistemas diferentes que se han denominado casos A, B, C y D. La Tabla I resume el binomio que forma cada combinación.

A la hora de comparar la bondad de estos sistemas se va a utilizar el factor de calidad (factor $Q$ ). El factor $Q$ se basa en una medida realizada directamente sobre el diagrama de ojo obtenido en un osciloscopio, a partir de $I_{1}$ e $I_{0}$

Tabla I

Combinaciones láser-fibra bajo estudio.

\begin{tabular}{||c|c|c||}
\hline Caso & DML & Fibra óptica \\
\hline A & DML-A & SMZ \\
\hline B & DML-A & NZ-DSF \\
\hline C & DML-T & SMZ \\
\hline D & DML-T & NZ-DSF \\
\hline
\end{tabular}
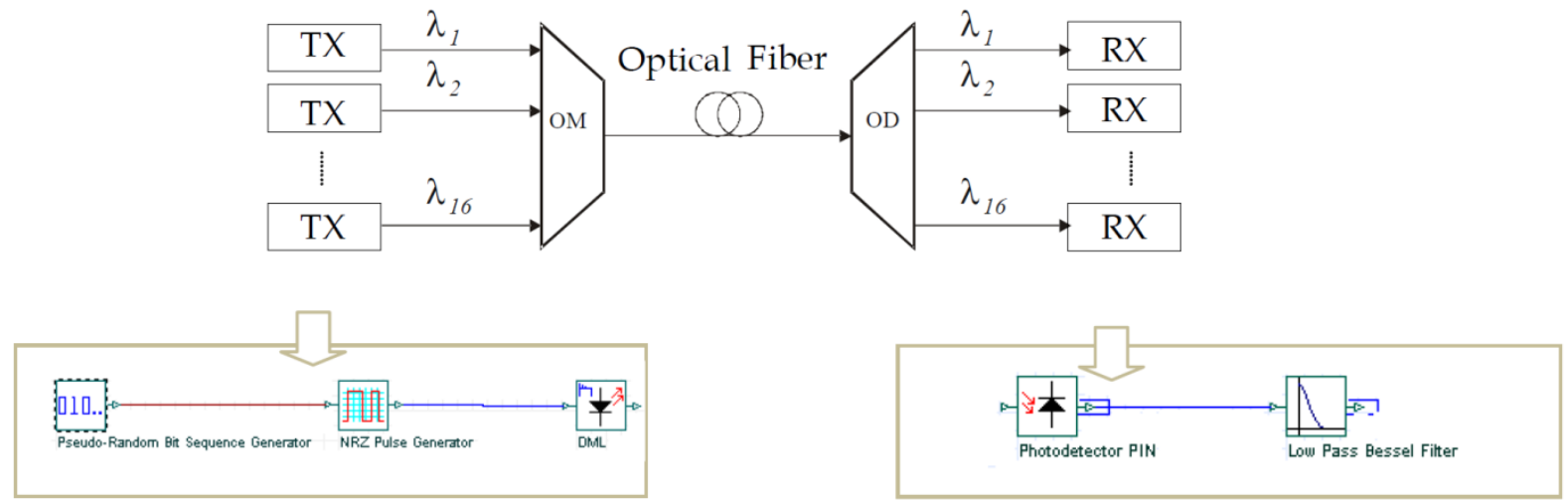

Fig. 12. Diagrama de bloques de sistema simulado. 
(niveles medios asociados a los niveles lógicos “1" y "0") y $\sigma_{1}, \sigma_{0}$ (desviaciones estándar para los niveles lógicos)

$$
Q=\frac{I_{1}-I_{0}}{\sigma_{1}-\sigma_{0}} .
$$

Este factor está relacionado con la tasa de error del sistema (BER) según la ecuación:

$$
B E R=\frac{1}{2} \operatorname{erfc}\left(\frac{Q}{\sqrt{2}}\right),
$$

siendo $\operatorname{erfc}(x)$ la función de error complementaria. Generalmente, se considera una transmisión libre de error para valores de $Q$ superiores a 7 (que se corresponde con valores de $B E R$ por debajo de $10^{-12}$ ).

Basándonos en este parámetro, podemos comparar los cuatro sistemas bajo estudio. La Fig. 13 resume los resultados obtenidos en las simulaciones realizadas para cada uno de los casos propuestos. En ella se ha representado el factor $Q$ para el canal correspondiente a una longitud de onda de $1551 \mathrm{~nm}$.

En todos los casos se ha variado la potencia de emisión de los DML para modificar la cantidad de chirp generado por el láser y el SPM provocado en la fibra. Se han simulado dos fibras de distintas características dispersivas para, de esta forma, evaluar el resultado de la interacción entre el chirp del láser y la dispersión de las fibras.

Del análisis de la Fig. 13, podemos extraer interesantes conclusiones. Lo primero a destacar es que todas las curvas presentan un máximo de calidad para un valor de potencia determinado [14], es decir, existe un valor de potencia óptimo, $P_{\text {optimo }}$, que conduce a un valor máximo de $Q$ para el canal considerado. Este valor óptimo

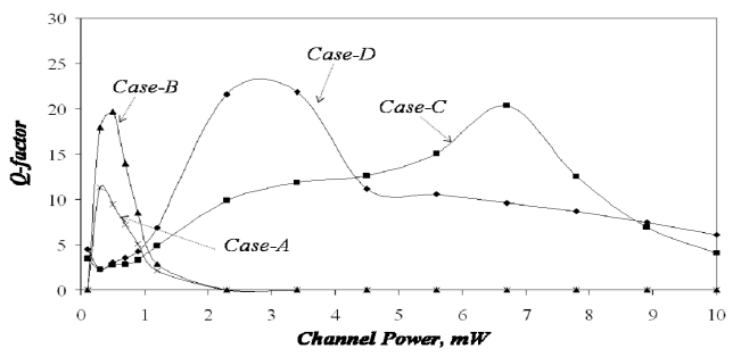

Fig. 13. Factor $Q$ en función de la potencia óptica del canal $1551 \mathrm{~nm}$, para los casos considerados en la Tabla I, tras atravesar $100 \mathrm{~km}$ de fibra con dispersión positiva o negativa [14]. coincide con el valor de potencia para el que se consigue el mejor balance entre los diferentes valores de chirp involucrados (DML, dispersión y SPM de la fibra). Por otra parte también se observa que el valor de $P_{\text {optimo }}$ depende de la combinación de elementos utilizados en cada caso. Estudiaremos las características propias de cada combinación.

\section{5.a. Casos A y B: transmisor adiabático dominante}

$\mathrm{Al}$ analizar el comportamiento de los Casos A y B, en los que se utilizan láseres con comportamiento adiabático dominante (DML-A), se observa que el valor de potencia para el que se alcanza el valor máximo de calidad está en torno a 0.3-0.46 $\mathrm{mW}$, independientemente del tipo de fibra. A partir de este valor la función empeora drásticamente al aumentar la potencia de salida del láser.

En ambos casos, el comportamiento adiabático dominante del DML provoca un desplazamiento de la frecuencia de emisión nominal en el bit 1 , dependiente de $P(t)$. Para valores superiores a $0.4 \mathrm{~mW}$, el desplazamiento que sufre el espectro es tal que los filtros existentes en el sistema (emplazados en los multiplexores y demultiplexores) empiezan a recortar parte del mismo, provocando el cierre del diagrama de ojo.

En la Fig. 14 se muestra el espectro de un DML con un comportamiento adiabático dominante junto a la función de transferencia del filtro óptico Gaussiano utilizado en la simulación.

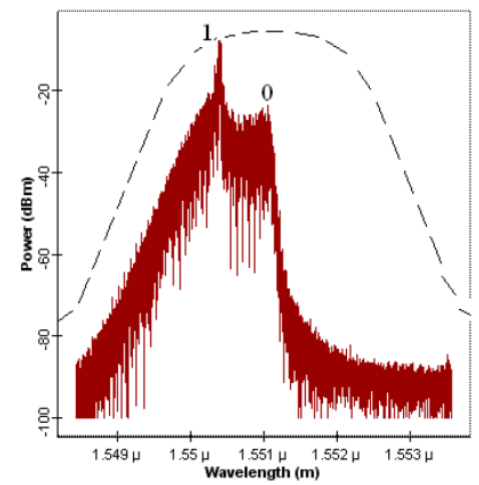

Fig. 14. Espectro del DML-A y respuesta de un filtro Gaussiano. 
Se observa que, debido a la pendiente del flanco de subida del filtro, el desplazamiento del espectro hacia el azul origina mayor atenuación en el pico de emisión del bit "1" que la producida sobre el pico de emisión del bit " 0 ". Esto produce un acercamiento entre los niveles de potencia del " 1 " y del " 0 ", es decir, una disminución del ratio de extinción que provoca un cierre en el diagrama de ojo y la disminución del factor $Q$. Este efecto es especialmente relevante en sistemas en anillo, en los que la señal óptica atraviesa una serie de filtros concatenados. El ancho de banda equivalente del conjunto de filtros es menor que el ancho de banda de cada filtro, por eso, cuanto mayor sea el número de filtros que atraviese la señal óptica, mayor será el cierre del diagrama de ojo producido [15].

Por otra parte, la forma de los pulsos ópticos que provienen de transmisores DML-A sufren una deformación al atravesar un medio dispersivo. En concreto, al propagarse por una fibra con dispersión positiva, la forma del pulso óptico muestra un overshoot en el flanco de subida que es proporcional a la potencia óptica. Esta deformación se produce en el flanco de bajada si la fibra presenta coeficiente de dispersión negativo [16].

A modo de ejemplo, la Fig. 16 muestra la evolución de la forma del pulso óptico de un DML-A cuando se varía su potencia de salida entre 0.5 a $4 \mathrm{~mW}$, después de atravesar $100 \mathrm{~km}$ de fibra NZDSF de dispersión negativa.

Esta falta de simetría en el pulso óptico genera el cierre del diagrama de ojo, disminuyendo la calidad del sistema en estas condiciones. La Fig. 17 muestra el diagrama de ojo del caso B (100 km de fibra NZ-DSF y DML
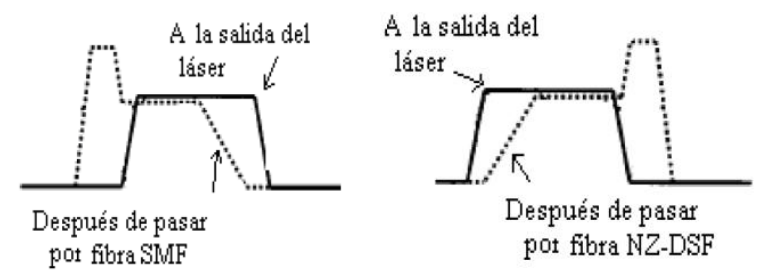

Fig. 15. Formas del pulso óptico de un láser DML al atravesar fibras de dispersión positiva (SMF) y negativa (NZ-DSF) [12].

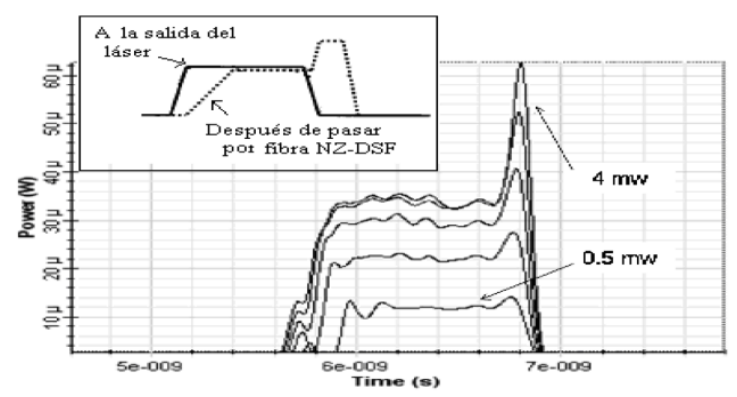

Fig. 16. Formas del pulso óptico de un láser DML-A a diferentes potencias después de atravesar $100 \mathrm{~km}$ de fibra de dispersión negativa.

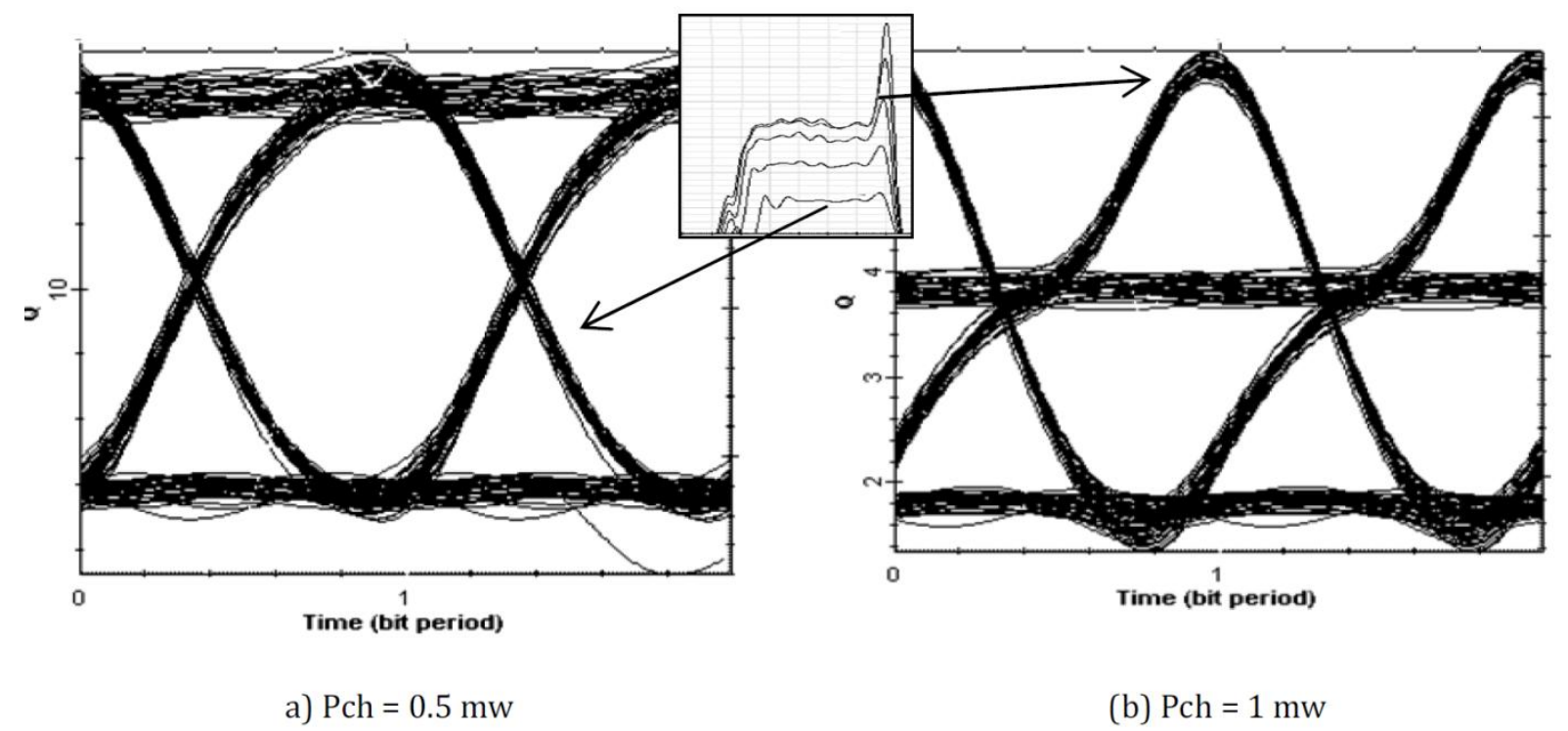

Fig. 17. Diagramas de ojo cuando la señal ha atravesado $100 \mathrm{~km}$ de fibra NZ-DSF con dispersión negativa y DML con comportamiento adiabático dominante emitiendo a dos potencias diferentes. 
con comportamiento adiabático dominante) para dos valores de potencias diferentes, (a) 0.5 mW y (b) $1 \mathrm{~mW}$, respectivamente. Para $0.5 \mathrm{~mW}$, como se puede observar en la Fig. 13, se obtiene un máximo de calidad en el sistema y el diagrama de ojo correspondiente (Fig. 17(a)) se muestra claramente abierto. Sin embargo, para potencias por encima de $1 \mathrm{~mW}$, la deformación de la forma del pulso se traslada al diagrama de ojo (Fig. 17(b)), generando importantes asimetrías y un cierre de más de $3 \mathrm{~dB}$.

\section{5.b. Casos C y D: transmisor transitorio dominante}

En los casos C y D, en los que se han simulado sistemas que emplean láseres con un comportamiento transitorio dominante (DMLT), como se puede observar en la Fig. 13, la potencia para la que se obtiene un máximo de calidad es $6.7 \mathrm{~mW}$ en el caso C (fibra con dispersión positiva) y $3 \mathrm{~mW}$ en el caso D (fibra con dispersión negativa).

En ambos casos el desplazamiento que se produce en el espectro de los DML-T cuando se aumenta la potencia de salida del láser es menor que en los casos A y B, permitiendo trabajar con valores de potencias superiores.

Para potencias de canal entre 0.1 y $4 \mathrm{~mW}$, el pulso óptico a la salida de la fuente se ve comprimido al atravesar una fibra con

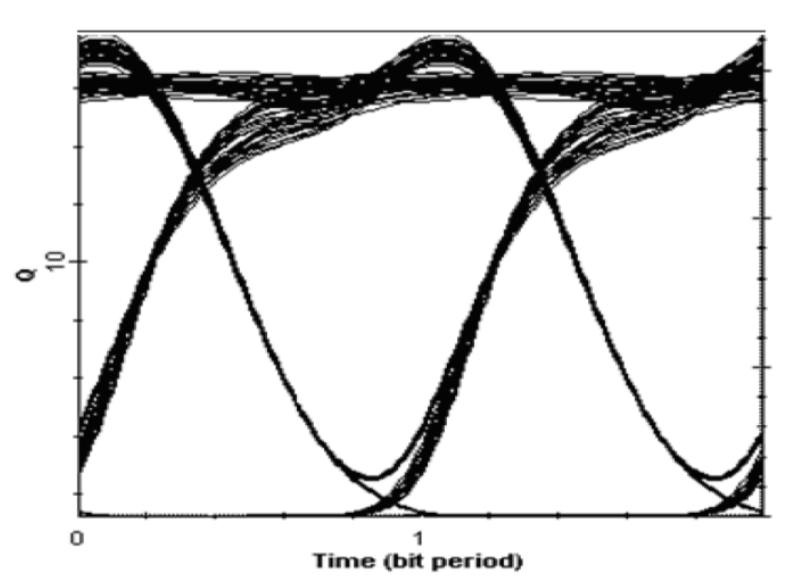

(a) Positiva dispersión negativa (caso D) y ensanchado si la dispersión tiene signo positivo (caso C). Por tanto, el comportamiento del sistema es mejor cuando se usan fibras de dispersión negativa.

Sin embargo, un aumento de potencia supone la aparición del fenómeno no lineal SPM. En este caso, el SPM puede colaborar compensando el efecto de la dispersión de las fibras con signos de dispersión positivos, de forma que, para potencias de canal entre 4 y $9 \mathrm{~mW}$, el caso $\mathrm{C}$ (DML-T/SMF) presenta mejor comportamiento que el D (DML-T/NZ-DSF). Para $P_{c h}$ superiores a $7 \mathrm{~mW}$, el aumento del chirp generado por el SPM deja de ser beneficioso en el balance total y el pulso se ensancha nuevamente disminuyendo la calidad del sistema.

En la Fig. 18 se muestra el diagrama de ojo para los casos C (a) y D (b) para una potencia media de salida de $7 \mathrm{~mW}$. El canal seleccionado es el centrado a $1551 \mathrm{~nm}$.

Si se comparan los diagramas de ojo de la Fig. 18 se observa, en el caso de la fibra con dispersión negativa (Fig. 18(b)), una interferencia entre símbolos y una falta de simetría que genera un diagrama de ojo mucho más cerrado que el caso de la fibra de dispersión positiva (Fig. 18(a)).

La conclusión más relevante extraída de este estudio es que existe una $P_{\text {optima }}$ que

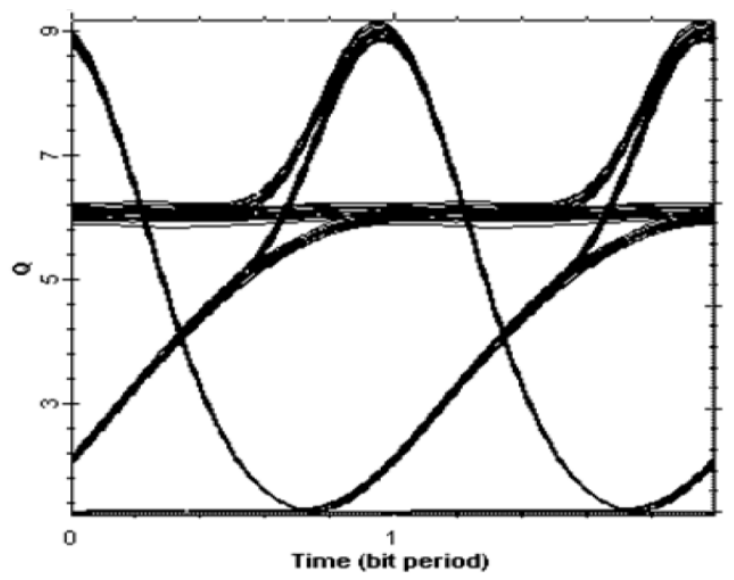

(b) Negativa

Fig. 18. Diagrama de ojo cuando se ha atravesado $100 \mathrm{~km}$ de fibra con dispersión positiva y negativa y se ha utilizado un láser con un comportamiento transitorio dominante emitiendo $7 \mathrm{~mW}$ de potencia. 
proporciona un balance óptimo entre los diferentes chirp involucrados y que permite que los sistemas que usan fibras con dispersión positiva tengan una calidad de transmisión similar a la obtenida con fibras de dispersión negativa, cuando se usan determinados láseres (con comportamiento transitorio dominante).

Este hecho es de particular importancia ya que evitaría el tener que modificar el tendido de las fibras existentes en la actualidad para conseguir los mismos criterios de calidad que los que se pueden obtener con fibras NZ-DSF.

\section{Gestión de la potencia óptica de emisión por canal para la mejora del factor $Q$}

La multiplexación CWDM es la tecnología usualmente empleada en entornos metro y de acceso ya que la transmisión de canales separados $20 \mathrm{~nm}$ entre sí, permite el uso de transmisores DML no refrigerados con un coste muy inferior a los láseres estabilizados y modulados externamente que exige el DWDM.

Al trabajar con tecnología CWDM hay que tener presente que cada canal se comportará de manera muy diferente, ya que la fibra exhibe una curva de dispersión y área efectiva que es función de la longitud de onda de trabajo. Así pues, se espera que la potencia óptima que consiga el mejor balance entre los distintos chirp, sea diferente para cada canal. Por tanto, para para completar este estudio hay que analizar cómo evoluciona la $P_{\text {optima }}$ en función del canal a tratar.

La Fig. 18 muestra el factor $Q$ en función de la potencia óptica por canal, obtenido en el sistema CWDM de la Fig. 12, formado por 16 canales. Por simplicidad sólo se han representado los canales centrados en 1531, 1551, 1571 y 1591 nm, respectivamente, y sólo se consideran los casos C (DMLT/SMF) (Fig. 19(a)) y D (DML-T/NZ-DSF) (Fig. 19(b)).

Del estudio realizado se pueden obtener diferentes conclusiones. Por una parte, se confirma, como era de esperar, que $P_{\text {optima }}$, es diferente para cada canal. Por ejemplo, en el caso D, para asegurar el máximo factor de calidad posible $\left(Q_{\max }\right)$ habría que fijar aproximada- mente $P_{c h}$ en $2.6 \mathrm{~mW}$ para el canal centrado en $1531 \mathrm{~nm} ; 2.8 \mathrm{~mW}$ para $1551 \mathrm{~nm} ; 3.1 \mathrm{~mW}$ para $1571 \mathrm{~nm}$ y $3.5 \mathrm{~mW}$ si el canal considerado es el centrado en $1591 \mathrm{~nm}$.

Dichos valores son a su vez diferentes si ahora el caso considerado es el caso C, para el que se obtendrían máximos de calidad ajustando la potencia de cada canal a $6.4(1531 \mathrm{~nm}), 6.7$ $\mathrm{mW}(1551 \mathrm{~nm}), 7.1 \mathrm{~mW}(1571 \mathrm{~nm})$ y $7.6 \mathrm{~mW}$ si el canal considerado es el centrado en $1591 \mathrm{~nm}$.

En la Fig. 20 se resumen los valores de la potencia óptima por canal $P_{c h}$, necesaria para alcanzar el máximo de calidad, en función de la dispersión total acumulada en el enlace. Los círculos blancos representan los casos en los que la dispersión en fibra es positiva y los círculos negros cuando es negativa. También se han marcado sobre la gráfica los puntos que corresponderían a los canales CWDM centrados en 1391 y $1551 \mathrm{~nm}$, después de recorrer $100 \mathrm{~km}$ de fibra SMF y NZ-DSF.

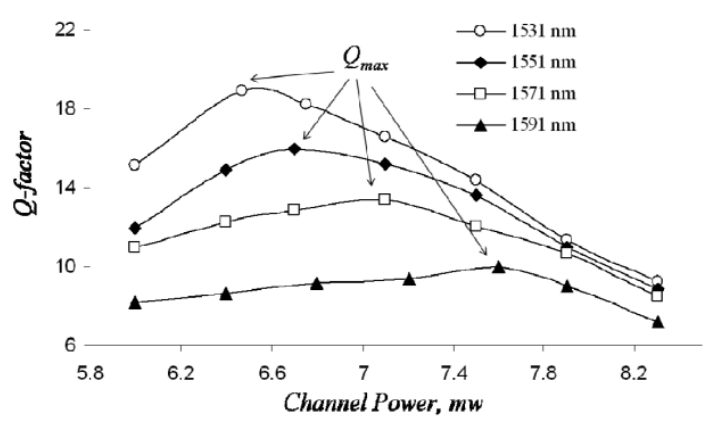

(a) Caso C (DML-T/SMF)

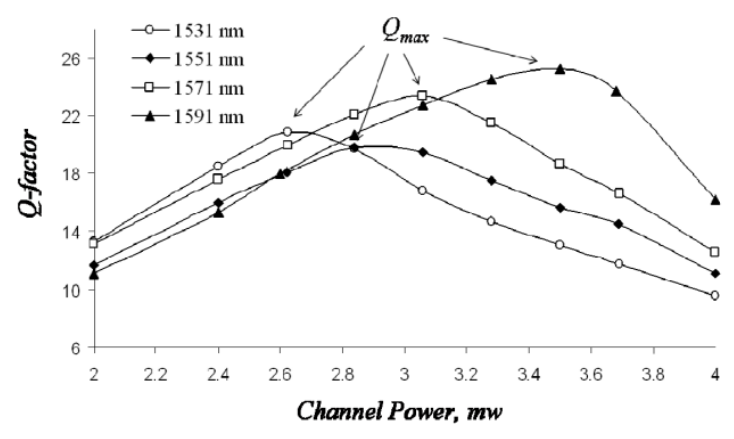

(b) Caso D (DML-T/NZ-DSF)

Fig. 19. Factor $Q$ versus $P_{c h}$ para los canales centrados en 1531, 1551, 1571 and $1591 \mathrm{~nm}$, respectivamente, para sistemas que usan combinaciones láser-fibra DML-T/SMF (a) and DML-T/NZ-DSF (b). 


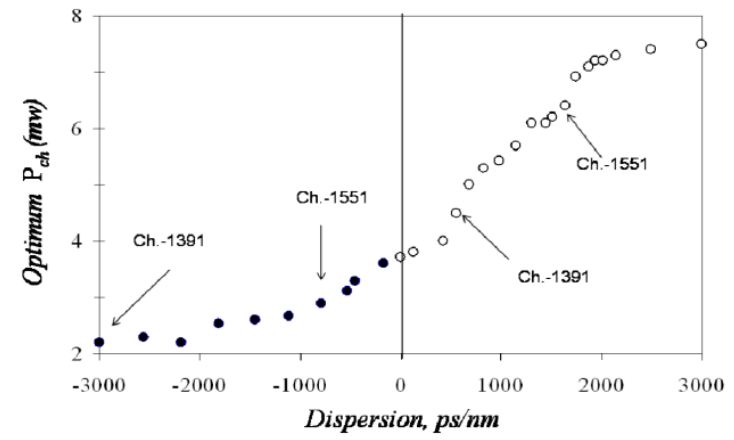

Fig. 20. Comparativa del valor óptimo de potencia versus la dispersión acumulada a lo largo del enlace, para una fibra de dispersión positiva (círculos blancos) y negativa (círculos negros).

Con la finalidad de facilitar el diseño de un sistema optimizado mediante este método, sería conveniente realizar una estimación, a priori, del valor de potencia a la que se debe fijar la salida del láser para cada uno de los canales. Para ello se ha desarrollado una expresión matemática, utilizando la herramienta de simulación Matlab, que se ajusta a la curva obtenida en la Fig. 19, donde se ha aproximado esta función a partir de una expresión polinómica de cuarto grado:

$$
f(x)=a x^{4}+b x^{3}+c x^{2}+d x+e,
$$

donde $x$ es la dispersión acumulada en el trayecto, y donde el mejor ajuste se ha obtenido con $a=-3.482 \times 10^{-14}, \quad b=-6.588 \times 10^{-11}$, $c=4.202 \times 10^{-7}, d=0.001435$ and $e=3.673$.

En la Fig. 21 se representa la función aproximada (línea continua) junto con los resultados obtenidos en la simulación (curva punteada), observándose una muy buena concordancia entre ellas.

Gracias a esta expresión se puede calcular, durante la etapa de prediseño, un valor de potencia óptica de partida de los DML que optimice el comportamiento del sistema, reduciendo el número de etapas de simulaciones necesarias para el diseño y por tanto el tiempo empleado en las mismas.

\section{Conclusiones}

El objeto de este trabajo ha sido la optimización de la transmisión de un enlace CWDM con topología punto a punto, identificando qué

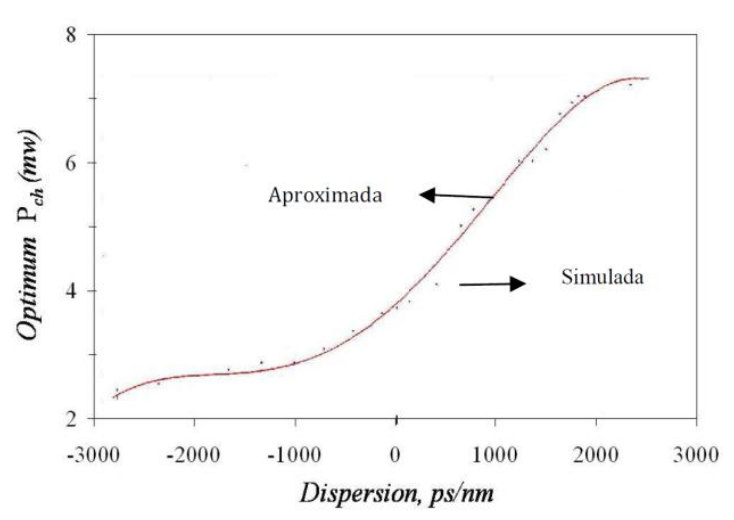

Fig. 21. Polinomio de cuarto grado que aproxima la curva que permite determinar a priori la potencia óptima de emisión en un sistema CWDM.

estrategia de diseño permite mejorar de forma sustancial la calidad de la transmisión y proponiendo técnicas económicamente viables.

Se ha demostrado la dependencia de $P_{c h^{-}}$ óptima con los parámetros del DML y la fibra empleada en el enlace. Así, seleccionando adecuadamente el transmisor, se ha demostrado que se pueden conseguir enlaces construidos a partir de fibras con dispersión positiva que presenten el mismo o mejor comportamiento que los que emplean fibras de dispersión negativa. Para ello se deben seleccionar láseres con comportamiento transitorio dominante $\mathrm{y}$ elegir una potencia de salida adecuada de forma que la interacción entre el chirp generado por las características dispersivas, tanto lineales como no-lineales, de la fibra y los efectos producidos por el chirp de los láseres modulados directamente se compensen entre ellos.

Se ha propuesto un método de cálculo que determina el valor de la potencia a la que debe fijarse cada canal CWDM para optimizar la transmisión, teniendo en cuenta que la dispersión de la fibra es función de la longitud de onda considerada. Este método consigue obtener la mejor de las respuestas de forma individualizada para cada uno de los canales que se multiplexan en la fibra, independientemente del modelo comercial de la misma y de la distancia del enlace, ya que se ha demostrado que el cálculo de la potencia óptima depende únicamente de la dispersión total acumulada a lo largo del enlace. Este método es de aplicación en cualquier sistema WDM. 